\title{
ASIAN OPTIONS UNDER ONE-SIDED LÉVY MODELS
}

\author{
P. PATIE, ${ }^{*}$ Université Libre de Bruxelles
}

\begin{abstract}
We generalize, in terms of power series, the celebrated Geman-Yor formula for the pricing of Asian options in the framework of spectrally negative Lévy-driven assets. We illustrate our result by providing some new examples.
\end{abstract}

Keywords: Asian option; Lévy process; exponential functional; hypergeometric-type function

2010 Mathematics Subject Classification: Primary 91G20; 60G51

\section{Introduction}

Asian options are path-dependent contingent claims whose settlement price is calculated with reference to the average price of the underlying security over a prescribed time period. In this paper we are concerned with the pricing of fixed-strike Asian call options in a market driven by a spectrally negative Lévy process, that is, a process with stationary and independent increments having no positive jumps. The motivation for studying such financial contracts in Lévy-driven asset models with no positive jumps is twofold. On the one hand, a commonly accepted remedy to the imperfections of the geometric Brownian motion as a model for asset prices is the use of exponential Lévy-type dynamics; see, e.g. [33]. Moreover, over the last years, it has been observed by several authors that the structure of the class of spectrally negative Lévy processes is relevant for modeling the dynamics of the prices of financial assets. For instance, Eberlein and Madan [14] provided a variety of economic reasons to support the consistency of processes with no positive jumps in the context of long-maturity stock price distributions embedded in option prices. Schoutens and Madan [25] also argued that spectrally negative Lévy processes are sufficient for long-dated options. In this regard, we mention that the markets for long-term options have witnessed an explosive growth over the last decade. Currently, liquid prices for maturities up to thirty years and beyond are shown for these type of products; see, e.g. [8]. On the other hand, as we will see in this paper, this class of models, including the Black-Scholes dynamics, is flexible and simple enough to provide a tractable expression for the Laplace transform with respect to time to maturity of the price of fixed-strike Asian options.

In this framework, it turns out that the issue of pricing Asian options is a great mathematical challenge. Indeed, it is already a difficult problem to determine the law of an additive functional of a diffusion process, such as the arithmetic average of the exponential of a Brownian motion, to be convinced that the case of Lévy processes might not be straightforward. This is probably one of the reasons why most of the literature studies focus only on the pricing of Asian options in Black-Scholes-type models.

Using stochastic calculus, and specifically the Bessel processes, Geman and Yor [17], see also the excellent monograph of Yor [37], obtained an analytical formula for the Laplace transform in time of the Asian option price. Their approach reveals that the issue of evaluating Asian options

Received 27 September 2011; revision received 31 August 2012.

* Postal address: Département de Mathématiques, Université Libre de Bruxelles, Boulevard du Triomphe, B-1050, Bruxelles, Belgique. Email address: ppatie@ulb.ac.be 
amounts to finding the law of the so-called exponential functional of the Brownian motion with drift taken at some independent exponential time. Then, many authors have been interested in characterizing the law of the exponential functional in the more general framework of Lévy processes. Beside some isolated cases and until very recently, only information regarding some transformations, such as the entire moments, or the tail behavior of the distribution has been identified; see, e.g. [5], [6], [19], and [26]. We refer the reader to the survey paper of Bertoin and Yor [3] for a nice description of these kinds of results. However, Patie [30] and [31] offered a power series and a contour integral representation of the law of this exponential functional for the class of spectrally negative Lévy processes. In this paper, relying on this result, we provide a generalization of the Geman-Yor formula in the context of spectrally negative Lévy processes.

Returning to the Black-Scholes framework, we mention that there is a substantial literature devoted to the issue of pricing Asian options. In particular, Rogers and Shi [32] formulated a one-dimensional partial differential equation that can model both floating and fixed strike Asian options. Donati-Martin et al. [10] expressed the prices of Asian options in terms of the resolvent density of some diffusions. We also note that Carr and Schröder [7] and, more recently, Schröder [35] used complex analysis techniques for inverting numerically or analytically the Geman-Yor Laplace transform. Dufresne [12], see also Schröder [34] and Linetsky [24], resorted to Laguerre polynomials for deriving an analytical expression for Asian call options. We also refer the reader to Fu et al. [16] for a description of numerical methods developed for approximating the price of these type of options in the Black-Scholes model. Beyond the diffusion case, we would like to mention that Večeř and $\mathrm{Xu}$ [36] provided an interesting formulation of Asian option prices in the general framework of special semimartingales as the solution of a boundary-value problem associated to a partial integrodifferential equation. Finally, the difficulty of getting analytical expressions for this problem have led many authors to finding some interesting upper and lower bounds for the prices of essentially discrete monitored Asian options. We refer the reader to [1] where such bounds are derived for implementing a static super hedge for fixed-strike Asian call options.

The remaining part of the paper is organized as follows. In the next section, after describing the financial market model, we discuss some basic ideas about the pricing of Asian call options. We also recall a recent result regarding the representation in terms of power series of the law of the exponential functional of spectrally negative Lévy processes. In Section 3 we state and prove the generalization of the Geman-Yor formula. Finally, we end the paper by providing three examples illustrating our main result. We also mention that parts of the results stated in Theorem 3.1 below were announced in the note [30].

\section{Preliminaries}

\subsection{The market model}

Let $\xi=\left(\xi_{t}\right)_{t \geq 0}$ be a spectrally negative Lévy process defined on a filtered probability space $\left(\Omega, \mathcal{F},\left(\mathcal{F}_{t}\right)_{t \geq 0}, \mathbb{P}\right)$, where $\left(\mathcal{F}_{t}\right)_{t \geq 0}$ is the filtration generated by $\xi$ satisfying the usual conditions. For any $x \in \mathbb{R}, \mathbb{P}_{x}$ stands for the law of $\xi$ when started at $x$, i.e. $\mathbb{P}_{x}$ is the law of $\xi+x$ under $\mathbb{P}=\mathbb{P}_{0}$. Accordingly, we will write $\mathbb{E}_{x}$ and $\mathbb{E}$ for the associated expectation operators. Next, we consider a financial market where two assets are traded. There is the riskless security whose price grows at the continuously compounding positive interest rate $r$. The dynamics of the risky asset $S=\left(S_{t}\right)_{t \geq 0}$ are governed by the exponential of $\xi$, that is, for any $t \geq 0$,

$$
S_{t}=\mathrm{e}^{\xi_{t}}
$$


We exclude the case when $\xi$ is degenerate, that is, when it is the negative of a subordinator, i.e. a process with increasing paths, or a pure drift process. In this setting, it is well known that the characteristic exponent $\Psi$, defined by

$$
\Psi(z)=\log \left(\mathbb{E}\left[\mathrm{e}^{\mathrm{i} z \xi_{1}}\right]\right), \quad z \in \mathbb{R},
$$

admits an analytical continuation to the lower half-plane and we set $\psi(u)=\Psi(-\mathrm{i} u), u \geq 0$. This means that, for any $u \geq 0, \psi$ admits the following Lévy-Khintchine representation:

$$
\psi(u)=\delta u+\frac{\sigma}{2} u^{2}+\int_{-\infty}^{0}\left(\mathrm{e}^{u y}-1-u y \mathbf{1}_{\{|y|<1\}}\right) m(\mathrm{~d} y) .
$$

Here $\sigma \geq 0$ is the Gaussian coefficient, $\delta \in \mathbb{R}$ is the drift, and the Lévy measure $m$ satisfies the integrability condition $\int_{-\infty}^{0}\left(1 \wedge y^{2}\right) m(\mathrm{~d} y)<\infty$. We refer the reader to the books of Bertoin [2] and Kyprianou [22] for backgrounds on Lévy processes. It is easily seen that the discounted asset price $\tilde{S}=\left(\tilde{S}_{t}=\mathrm{e}^{-r t} S_{t}\right)_{t \geq 0}$ is also a spectrally negative Lévy process with Laplace exponent $\tilde{\psi}(u)=\psi(u)-r u$. Note that, by changing the value of the drift, we may also consider the case when the risky asset pays a continuous compound dividend yield at some fixed rate per annum. Next, we recall that the fundamental theorem of asset pricing, see [9], requires that $\tilde{S}$ is a (local) martingale under a probability measure which is equivalent to the historical one. For the sake of simplicity, by assuming that

$$
\psi(1)=r
$$

we set $\mathbb{P}$ to be a risk-neutral probability measure. We mention that Eberlein et al. [15] gave a complete description of the set of equivalent local martingale measures in the setting of Lévy-driven assets.

Let us now recall some basic properties of the Laplace exponent $\psi$ which will be useful in the sequel. First, $\psi$ is continuous on $\mathbb{R}^{+}$with $\psi(0)=0$ and $\lim _{u \rightarrow \infty} \psi(u)=+\infty$. By monotone convergence, we obtain $\mathbb{E}\left[\xi_{1}\right]=\psi^{\prime}\left(0^{+}\right)=\delta+\int_{-\infty}^{-1} y m(\mathrm{~d} y) \in[-\infty, \infty)$. Let us write

$$
R=\lim _{u \rightarrow \infty} \frac{\psi(u)}{u} .
$$

Then, $R$ may take different values depending upon the coefficients of $\psi$. Indeed, if $\sigma=0$ and $\int_{-\infty}^{0} 1 \wedge|y| m(\mathrm{~d} y)<\infty$, that is, the Lévy process $\xi$ has paths of bounded variation, then we obtain, see [2, Corollary VII.5], $R=\bar{\delta}$ with

$$
\bar{\delta}=\delta-\int_{-1}^{0} y m(\mathrm{~d} y) .
$$

Since we have excluded the degenerate cases, it is plain that $\bar{\delta}>0$. For the other cases, $R=+\infty$. To summarize, we have

$$
R= \begin{cases}\bar{\delta} & \text { if } \sigma=0 \text { and } \int_{-\infty}^{0} 1 \wedge|y| m(\mathrm{~d} y)<\infty \\ \infty & \text { otherwise }\end{cases}
$$

Note that 0 is always a root of the equation $\psi(u)=0$. However, in the case $\mathbb{E}\left[\xi_{1}\right]<0$, this equation admits another positive root, which we denote by $\theta$. Moreover, for any $\mathbb{E}\left[\xi_{1}\right] \in[-\infty, \infty)$, the function $u \mapsto \psi(u)$ is continuous and increasing on $[\max (\theta, 0), \infty)$. Thus, it has a well-defined inverse function $\phi:[0, \infty) \rightarrow[\max (\theta, 0), \infty)$ which is also continuous and increasing. 


\subsection{Asian options}

Let us start by introducing the so-called exponential functional of the Lévy process $\xi$ which is defined, for any $0 \leq t_{0} \leq t$, by

$$
\Sigma_{t_{0}, t}=\int_{t_{0}}^{t} \mathrm{e}^{\xi_{s}} \mathrm{~d} s
$$

Next, we set, for any $t_{0}, t \geq 0$,

$$
\bar{\Sigma}_{t_{0}, t}=\frac{\Sigma_{t_{0}, t}}{t-t_{0}} .
$$

We simply write $\Sigma_{t}=\Sigma_{0, t}$ and $\bar{\Sigma}_{t}=\bar{\Sigma}_{0, t}$. The payoff of the arithmetic Asian call option written at time $t_{0}>0$, with maturity $T>0$ and fixed strike price $K$, is given by

$$
\left(\bar{\Sigma}_{t_{0}, T}-K\right)_{+}
$$

By an arbitrage argument, the value at time $t$ of the Asian call option is

$$
C_{t}\left(t_{0}, T\right)=\mathrm{e}^{-r(T-t)} \mathbb{E}_{x}\left[\left(\bar{\Sigma}_{t_{0}, T}-K\right)_{+} \mid \mathcal{F}_{t}\right] .
$$

In the Black-Scholes model, Geman and Yor [18] showed that this conditional expectation could be factorized into simple terms. In what follows, we state the extension of their result to the general framework of Lévy processes whose proof is straightforward.

Proposition 2.1. Let us assume that $\psi(1)=r$. Then, for any $t_{0} \leq t<T$, we have

$$
C_{t}\left(t_{0}, T\right)=\frac{\mathrm{e}^{-r(T-t)}}{T-t_{0}} S_{t} \mathbb{E}\left[\left(\widehat{\Sigma}_{T-t}-K^{\prime}\right)_{+}\right],
$$

where $\widehat{\Sigma}_{T-t}$ is a copy of $\Sigma_{T-t}$ independent of $\mathcal{F}_{t}$ and

$$
K^{\prime}=\frac{K\left(T-t_{0}\right)-\Sigma_{t_{0}, t}}{S_{t}} .
$$

A direct consequence of Proposition 2.1 is that the price of an Asian option depends on the first moment of the random variable $\left(\Sigma_{t}-K^{\prime}\right)_{+}$. Unfortunately, it is a challenging mathematical problem to derive a tractable expression for this quantity. Instead, Geman and Yor [18] suggested computing such a moment, but for the exponential functional considered at some random time. More precisely, by replacing the time-dependent strike $K^{\prime}$ by a constant $a>0$, we consider the function

$$
\mathbb{E}\left[\left(\Sigma_{e_{q}}-a\right)_{+}\right]=q \int_{0}^{\infty} \mathrm{e}^{-q t} \mathbb{E}\left[\left(\Sigma_{t}-a\right)_{+}\right] \mathrm{d} t,
$$

where $e_{q}$ is an exponentially distributed random variable of parameter $q>0$, taken independent of $\xi$. The value of the option is then obtained by inverting the above Laplace transform in time and by choosing $a=K^{\prime}$.

\subsection{Law of the exponential functional}

It is now clear that to generalize the Geman-Yor formula to spectrally negative Lévy processes one has to compute the first truncated moment of the random variable $\Sigma_{e_{q}}$. In this part, we recall a recent result obtained by Patie [30], [31] regarding the distribution of this positive random variable. To this end, we proceed by introducing some notation taken 
from [29]. First, let $\psi$ be of the form (2.1) with $\psi^{\prime}\left(0^{+}\right) \geq 0$. Then, set $a_{0}=1$ and, for any $n=1,2, \ldots$,

$$
a_{n}(\psi)=\left(\prod_{k=1}^{n} \psi(k)\right)^{-1}
$$

In [29], the author introduced the power series

$$
\tau_{\psi}(z)=\sum_{n=0}^{\infty} a_{n}(\psi) z^{n}
$$

and showed by means of classical criteria that the mapping $z \mapsto \mathcal{I}_{\psi}(z)$ is an entire function. Note that the condition $\psi^{\prime}\left(0^{+}\right) \geq 0$ implies that all of the coefficients in the definition of $\tau_{\psi}(z)$ are strictly positive. We refer the reader to [29] for interesting analytical properties enjoyed by these power series and also for connections with well-known special functions, such as, for instance, the modified Bessel functions and several generalizations of the Mittag-Leffler function. Next, let $G_{\rho}$ be a random variable having the gamma distribution with parameter $\rho>0$, that is, its distribution is given by $g(\mathrm{~d} t)=\left(\mathrm{e}^{-t} t^{\rho-1} / \Gamma(\rho)\right) \mathrm{d} t, t>0$, with $\Gamma$ the Euler gamma function. Then, in [28], the author suggested the generalization

$$
\mathcal{I}_{\psi}(\rho ; z)=\mathbb{E}\left[\mathcal{I}_{\psi}\left(G_{\rho} z\right)\right]=\frac{1}{\Gamma(\rho)} \int_{0}^{\infty} \mathrm{e}^{-t} t^{\rho-1} \mathcal{L}_{\psi}(t z) \mathrm{d} t
$$

By means of the integral representation of the gamma function $\Gamma(\rho)=\int_{0}^{\infty} \mathrm{e}^{-t} t^{\rho-1} \mathrm{~d} t$, $\operatorname{Re}(\rho)>0$, see, e.g. [23, Chapter 1], and an argument of dominated convergence, we obtain the power series representation

$$
\tau_{\psi}(\rho ; z)=\frac{1}{\Gamma(\rho)} \sum_{n=0}^{\infty} a_{n}(\psi) \Gamma(\rho+n) z^{n}
$$

which is easily seen to be valid for any $|z|<R$, where we recall that $R$ is defined in (2.2). Moreover, for any $|z|<R$, the mapping $\rho \mapsto I_{\psi}(\rho ; z)$ is a meromorphic function defined for all complex numbers $\rho$ except at the poles of the gamma function, that is, at the points $\rho=0,-1, \ldots$ However, they are removable singularities. Indeed, for any $|z|<R$ and any integer $N \in \mathbb{N}$, we have, by means of the recurrence relation $\Gamma(z+1)=z \Gamma(z), \tau_{\psi}(0 ; z)=1$ and

$$
\tau_{\psi}(-N ; z)=\sum_{n=0}^{N}(-1)^{n} \frac{\Gamma(N+1)}{\Gamma(N+1-n)} a_{n}(\psi) z^{n} .
$$

Thus, by uniqueness of the analytical extension for any $|z|<R, \mathcal{I}_{\psi}(\rho ; z)$ is an entire function in $\rho$. Note also that, for $\rho=0,-1, \ldots$, as a polynomial, $\tau_{\psi}(-\rho ; z)$ is an entire function in $z$. In the following, we recall a result from [31] which summarizes the above claims and provide an analytical continuation of $\mathcal{I}_{\psi}(\rho ; z)$ in the case $R=\bar{\delta}$, that is, when $\xi$ is with paths of bounded variation.

Proposition 2.2. ([31].) 1 . If $R=\infty$ then $\mathcal{I}_{\psi}(\rho ; z)$ is an entire function in both arguments $z$ and $\rho$. 
2. If $R=\bar{\delta}$ then $\mathcal{I}_{\psi}(\rho ; z)$ is analytic in the disc $|z|<\bar{\delta}$ and, for any fixed $\rho=0,-1, \ldots$, $\tau_{\psi}(\rho ; z)$, as a polynomial, is an entire function. Moreover, for any $\rho \in \mathbb{C}, \tau_{\psi}(\rho ; z)$ admits, in the half-plane $\operatorname{Re}(z)<\bar{\delta} / 2$, the power series representation

$$
\tau_{\psi}(\rho ; z)=\left(1-\frac{z}{\bar{\delta}}\right)^{-\rho} \sum_{n=0}^{\infty} \tau_{\psi}(-n ; \bar{\delta}) \frac{\Gamma(\rho+n)}{\Gamma(\rho) n !}\left(\frac{z}{z-\bar{\delta}}\right)^{n}
$$

Finally, for any fixed $\operatorname{Re}(z)<\bar{\delta} / 2, \tau_{\psi}(\rho ; z)$ is an entire function in the argument $\rho$.

We mention that a representation as a contour integral of the function $\mathcal{I}_{\psi}(\rho ; z)$ is given in Appendix A. Next, we write, for any $q>0$,

$$
\gamma=\phi(q)
$$

and we set

$$
\psi_{\gamma}(u)=\psi(u+\gamma)-q, \quad u, q \geq 0
$$

$\psi_{\gamma}$ is well known to be the Laplace exponent of the so-called Esscher transform of $\xi$. Thus, it is again the Laplace exponent of a spectrally negative Lévy process. Moreover, we have $\psi_{\gamma}^{\prime}\left(0^{+}\right)=\psi^{\prime}(\gamma)=1 / \phi^{\prime}(q)>0$ since $\phi$ is the Laplace exponent of a subordinator and, hence, it is an increasing function. We are now ready to state the following result which provides an expression for the law of $\Sigma_{e_{q}}$.

Theorem 2.1. ([30], [31, Theorem 2.1].) Let $q>0$. Then there exists a constant $C_{\gamma}>0$ such that

$$
\tau_{\psi_{\gamma}}(\gamma ;-x) \sim \frac{x^{-\gamma}}{C_{\gamma}} \quad \text { as } x \rightarrow \infty .
$$

(Here $f(x) \sim g(x)$ as $x \rightarrow$ a means that $\lim _{x \rightarrow a} f(x) / g(x)=1$ for any $a \in[0, \infty]$.) Moreover, the law of $\Sigma_{e_{q}}$ under $\mathbb{P}$ is absolutely continuous with a density, denoted by $s_{\gamma}$, given by

$$
s_{\gamma}(t)=\gamma C_{\gamma} t^{-\gamma-1} \mathcal{I}_{\psi_{\gamma}}\left(1+\gamma ;-t^{-1}\right), \quad t>0 .
$$

Remark 2.1. If we assume that $\psi^{\prime}\left(0^{+}\right)<0$, which is equivalent, from the strong law of large numbers for Lévy processes, to $\lim _{t \rightarrow \infty} \xi_{t}=-\infty$ almost surely, then we have $\lim _{q \rightarrow 0} \phi(q)=$ $\theta>0$, where we recall that $\psi(\theta)=0$. Under this condition, the perpetual exponential functional $\Sigma_{\infty}=\int_{0}^{\infty} \mathrm{e}^{\xi_{s}} \mathrm{~d} s$ is well defined and its density, denoted by $s_{\theta}$, is given by

$$
s_{\theta}(t)=\lim _{q \rightarrow 0} s_{\phi(q)}(t), \quad t>0 .
$$

The expression of $s_{\theta}(t)$ can be found in [31, Theorem 2.1] and generalizes a result of Dufresne [11] obtained in the case of the Brownian motion with a negative drift.

The proof of the theorem is rather technical but the main steps can be described as follows. First, we use the Lamperti mapping which allows us to connect the law of the exponential functional $\Sigma_{e_{q}}$ to the law of the absorption time of a positive self-similar Markov process generalizing the Bessel processes. Then, by means of the self-similarity property, we show that the law of this latter stopping time is related to the probability that the absorption time of an associated transient Ornstein-Uhlenbeck process is finite, which turns out to be a quantity much easier to compute. Let us mention that such devices hold in the framework of two-sided Lévy processes. Finally, we derive an expression for this probability by combining complex analysis techniques with fluctuation identities for positive self-similar Markov processes obtained recently in [28] and [29]. The extension of this part of the proof to more general 
Lévy processes seems difficult. Indeed, assuming that the process has two-sided jumps but admits all positive exponential moments, which implies the existence of a Laplace exponent $\psi$, then it is a difficult matter, if true, to show that the mapping $\tau_{\psi_{\gamma}}\left(1+\gamma ;-t^{-1}\right)$ is nonnegative valued for any $t>0$, which is a necessary condition for (2.7) to be a density.

\section{A generalized Geman-Yor formula}

According to Proposition 2.1, the pricing of Asian options in the framework of Lévy processes amounts to computing the first moment of the random variable $\left(\Sigma_{t}-K\right)_{+}$. As already discussed in the previous section, this is a difficult task and instead we compute, for any $K>0$, the functional

$$
\mathbb{E}\left[\left(\Sigma_{e_{q}}-K\right)_{+}\right]
$$

where we recall that $e_{q}$ is an exponentially distributed random variable of parameter $q>0$ which is taken independent of $\xi$. We now state the generalization of the Geman-Yor formula to spectrally negative Lévy processes.

Theorem 3.1. For any $K>0$ and $q>\psi(1)$, we have

$$
\mathbb{E}\left[\left(\Sigma_{e_{q}}-K\right)_{+}\right]=\frac{C_{\gamma}}{\gamma-1} K^{1-\gamma} \tau_{\psi_{\gamma}}\left(\gamma-1 ;-K^{-1}\right) .
$$

Proof. Let us first consider the Mellin transform of the positive random variable $\left(\Sigma_{e_{q}}-K\right)_{+}$, which is defined, for $\kappa \in \mathrm{i} \mathbb{R}$, the imaginary line, by

$$
\mathcal{M}(\kappa)=\mathbb{E}\left[\left(\Sigma_{e_{q}}-K\right)_{+}^{-\kappa}\right] .
$$

It is plain, if both quantities exist, that

$$
\mathbb{E}\left[\left(\Sigma_{e_{q}}-K\right)_{+}\right]=\mathcal{M}(-1) .
$$

Next, let us write $\gamma=\phi(q)$ and, for any integer $N$,

$$
\mathcal{M}^{N}(\kappa)=\int_{0}^{\infty}(t-K)_{+}^{-\kappa} s_{\gamma}^{N}(t) \mathrm{d} t,
$$

where $s_{\gamma}^{N}(t)=\gamma C_{\gamma} t^{-\gamma-1} \ell_{\psi_{\gamma}}^{N}\left(\gamma ;-t^{-1}\right)$ and $\ell_{\psi_{\gamma}}^{N}(\gamma ; z)$ is the power series $\ell_{\psi_{\gamma}}(\gamma ; z)$ truncated at order $N$. Now, we split the proof of identity (3.1) into two parts.

First, we consider the case when $R=\infty$. From (2.4), we have, for any integer $N$,

$$
\begin{aligned}
\mathcal{M}^{N}(\kappa) & =\int_{K}^{\infty}(t-K)^{-\kappa} s_{\gamma}^{N}(t) \mathrm{d} t \\
& =\frac{C_{\gamma}}{\Gamma(\gamma)} \sum_{n=0}^{N}(-1)^{n} a_{n}\left(\psi_{\gamma}\right) \Gamma(\gamma+1+n) \int_{K}^{\infty}\left(1-\frac{K}{t}\right)^{-\kappa} t^{-n-\kappa-\gamma-1} \mathrm{~d} t,
\end{aligned}
$$

where we have used the recurrence formula of the gamma function $\Gamma(z+1)=z \Gamma(z)$, $\operatorname{Re}(z)>0$. Next, performing the change of variable $v=K / t$, we obtain

$$
\begin{aligned}
\mathcal{M}^{N}(\kappa) & =\frac{C_{\gamma}}{\Gamma(\gamma)} \sum_{n=0}^{N}(-1)^{n} a_{n}\left(\psi_{\gamma}\right) \Gamma(\gamma+1+n) K^{-n-\kappa-\gamma} \int_{0}^{1}(1-v)^{-\kappa} v^{\gamma+\kappa+n-1} \mathrm{~d} v \\
& =\frac{C_{\gamma} \Gamma(1-\kappa)}{\Gamma(\gamma)} K^{-\kappa-\gamma} \sum_{n=0}^{N} a_{n}\left(\psi_{\gamma}\right)(-K)^{-n} \Gamma(\gamma+\kappa+n),
\end{aligned}
$$


where the last line follows from the integral representation of the beta function, see, e.g. [20, Formula 3.191(1)],

$$
\frac{\Gamma(x) \Gamma(y)}{\Gamma(x+y)}=\int_{0}^{1}(1-v)^{x-1} v^{y-1} \mathrm{~d} v, \quad \operatorname{Re}(x), \operatorname{Re}(y)>0 .
$$

By the principle of analytical continuation, we deduce that identity (3.2) is valid in the strip $\wp_{\gamma}=\{\kappa \in \mathbb{C} ;-\gamma<\operatorname{Re}(\kappa)<1\}$. Next, we have, for any $\kappa \in \varsigma_{\gamma}$,

$$
\lim _{N \rightarrow \infty} \mathcal{M}^{N}(\kappa)=\frac{C_{\gamma} \Gamma(\gamma+\kappa) \Gamma(1-\kappa)}{\Gamma(\gamma)} K^{-\kappa-\gamma} \ell_{\psi_{\gamma}}\left(\gamma+\kappa ;-K^{-1}\right) .
$$

With the function on the right-hand side of this equality being holomorphic on the positive half-plane, we deduce by an argument of dominated convergence, see, e.g. [27, Theorem 8.1, Chapter 2], that, for any $\operatorname{Re}(K)>0$ and $\kappa \in \varsigma_{\gamma}$,

$$
\mathcal{M}(\kappa)=\frac{C_{\gamma} \Gamma(\gamma+\kappa) \Gamma(1-\kappa)}{\Gamma(\gamma)} K^{-\kappa-\gamma} \ell_{\psi_{\gamma}}\left(\gamma+\kappa ;-K^{-1}\right) .
$$

Moreover, since $\phi$ is increasing on $\mathbb{R}^{+}$, our assumption leads to the condition $\gamma>1$. Hence, by resorting again to the principle of analytical continuation and using the recurrence relation of the gamma function, we obtain

$$
\mathbb{E}\left[\left(\Sigma_{e_{q}}-K\right)_{+}\right]=\frac{C_{\gamma}}{\gamma-1} K^{1-\gamma} \ell_{\psi_{\gamma}}\left(\gamma-1 ;-K^{-1}\right),
$$

which proves our claim in the case $R=\infty$.

Next, assuming that $R=\bar{\delta}<\infty$, where we recall that $\bar{\delta}$ is defined in (2.3), and keeping the same notation as above, we have, from (2.5),

$$
\begin{aligned}
\mathcal{M}^{N}(\kappa)= & \int_{K}^{\infty}(t-K)^{-\kappa} s_{\gamma}^{N}(t) \mathrm{d} t \\
= & C_{\gamma} \sum_{n=0}^{N} \tau_{\psi_{\gamma}}(-n ; \bar{\delta}) \frac{\Gamma(\gamma+1+n)}{n ! \Gamma(\gamma)} \\
& \quad \times \int_{K}^{\infty} t^{-(\gamma+\kappa+n+1)}\left(1-\frac{K}{t}\right)^{-\kappa}\left(1+\frac{1}{\bar{\delta} t}\right)^{-(\gamma+1+n)} \mathrm{d} t \bar{\delta}^{-n} \\
= & C_{\gamma} K^{-\gamma-\kappa} \sum_{n=0}^{N} \tau_{\psi_{\gamma}}(-n ; \bar{\delta}) \frac{\Gamma(\gamma+1+n)}{n ! \Gamma(\gamma)} \\
& \quad \times \int_{0}^{1} v^{\gamma+\kappa+n-1}(1-v)^{-\kappa}\left(1+\frac{v}{\bar{\delta} K}\right)^{-(\gamma+1+n)} \mathrm{d} v(\bar{\delta} K)^{-n},
\end{aligned}
$$

where we have performed the change of variable $v=u / K$. Next, by means of the identity, which is found in [20, Formula 3.197(4)],

$\frac{\Gamma(x) \Gamma(y)}{\Gamma(x+y)}(1+a)^{-x}=\int_{0}^{1}(1-v)^{x-1} v^{y-1}(1+a v)^{-x-y} \mathrm{~d} v, \quad \operatorname{Re}(x), \operatorname{Re}(y)>0, a>-1$, 
we deduce that, for any $K>0$ and $\kappa \in \varsigma_{\gamma}$,

$$
\begin{aligned}
\mathcal{M}^{N}(\kappa) & =\frac{C_{\gamma} K^{-\gamma-\kappa} \Gamma(1-\kappa)}{\Gamma(\gamma)} \sum_{n=0}^{N} \tau_{\psi_{\gamma}}(-n ; \bar{\delta}) \frac{\Gamma(\gamma+\kappa+n)}{n !}(\bar{\delta} K)^{-n}\left(1+\frac{1}{\bar{\delta} K}\right)^{-(\kappa+\gamma+n)} \\
& =\frac{C_{\gamma} K^{-\gamma-\kappa} \Gamma(1-\kappa)}{\Gamma(\gamma)}\left(1+\frac{v}{\bar{\delta} K}\right)^{-\gamma-1} \sum_{n=0}^{N} \tau_{\psi_{\gamma}}(-n ; \bar{\delta}) \frac{\Gamma(\gamma+\kappa+n)}{n !}(1+\bar{\delta} K)^{-n} \\
& =\frac{C_{\gamma} K^{-\gamma-\kappa} \Gamma(1-\kappa) \Gamma(\gamma+\kappa)}{\Gamma(\gamma)} l_{\psi_{\gamma}}^{N}\left(\gamma+\kappa ;-K^{-1}\right) .
\end{aligned}
$$

Hence, we get, by dominated convergence, for any $K>0$ and $\kappa \in \varsigma_{\gamma}$,

$$
\mathcal{M}(\kappa)=\frac{C_{\gamma} \Gamma(\gamma+\kappa) \Gamma(1-\kappa)}{\Gamma(\gamma)} K^{-\kappa-\gamma} \ell_{\psi_{\gamma}}\left(\gamma+\kappa ;-K^{-1}\right) .
$$

The proof of the theorem is then completed by following a line of reasoning similar to the previous case.

Remark 3.1. 1. As observed by Geman and Yor [18] in the Black-Scholes model, one can also easily compute the value of the Asian call option under the Lévy model in the case where the strike $K$ is nonpositive. Indeed, we have

$$
C_{0}(0, T)=\mathrm{e}^{-r T}\left(S_{0} \int_{0}^{T} \mathbb{E}\left[\mathrm{e}^{\xi_{s}}\right] \mathrm{d} s-K\right)=\frac{1}{r}\left(1-\mathrm{e}^{-r T}\right) S_{0}-\mathrm{e}^{-r T} K,
$$

where we recall that $\psi(1)=r$.

2. By means of the symmetry relationship, established by Henderson and Wojakowski [21] in the Black-Scholes model, see also Eberlein and Papapantoleon [13] for its extension to Lévy process markets, between floating-strike and fixed-strike Asian options for assets driven, one could also derive from the previous result the price of the floating-strike Asian put option.

\section{Examples}

\subsection{The Black-Scholes model revisited}

We first consider the case when $S$ follows the Black-Scholes dynamics. That is, under the unique risk-neutral probability measure $\mathbb{P}, \xi$ is given, for any $t \geq 0$, by

$$
\xi_{t}=\sigma B_{t}+\delta t
$$

where $B=\left(B_{t}\right)_{t \geq 0}$ is a standard Brownian motion, $\sigma>0$, and $\delta=r-\sigma^{2} / 2$. It is plain that

$$
\psi(u)=\frac{\sigma^{2}}{2} u^{2}+\delta u, \quad u \geq 0,
$$

and $\psi(1)=r$. Next, we observe that, for any $q>0$,

$$
\phi(q)=\frac{\sqrt{2}}{\sigma}\left(\sqrt{q+\frac{\delta^{2}}{2 \sigma^{2}}}-\frac{\delta}{\sqrt{2} \sigma}\right) .
$$


Thus,

$$
\psi_{\gamma}(u)=\frac{\sigma^{2}}{2} u^{2}+\left(\sigma^{2} \gamma+\delta\right) u, \quad u \geq 0 .
$$

Moreover, setting $b=2 \gamma+2 \delta / \sigma^{2}$, we have, for any $n \geq 1$,

$$
a_{n}\left(\psi_{\gamma}\right)^{-1}=\prod_{k=1}^{n} \psi_{\gamma}(k)=\frac{\sigma^{2 n}}{2^{n}} n ! \prod_{k=1}^{n} k+b=\frac{\sigma^{2 n} \Gamma(n+b+1)}{2^{n} \Gamma(b+1)} n ! .
$$

Since $R=\infty$, we have, for any $z, \rho \in \mathbb{C}$,

$$
\tau_{\psi_{\gamma}}(\rho ; z)=\frac{\Gamma(b+1)}{\Gamma(\rho)} \sum_{n=0}^{\infty} \frac{\Gamma(\rho+n)}{n ! \Gamma(n+b+1)}\left(-\frac{2 z}{\sigma^{2}}\right)^{n}=\Phi\left(\rho, b+1 ;-\frac{2 z}{\sigma^{2}}\right),
$$

where $\Phi$ stands for the confluent hypergeometric function. We refer the reader to [23, Chapter 9] for useful properties of this function. Next, using the asymptotic

$$
\Phi(\rho, b+1 ;-x) \sim \frac{\Gamma(b+1)}{\Gamma(b+1-\rho)} x^{-\rho} \quad \text { as } x \rightarrow \infty,
$$

we get, from (2.6),

$$
C_{\gamma}=\frac{\Gamma(b+1-\gamma)}{\Gamma(b+1)}\left(\frac{2}{\sigma^{2}}\right)^{\gamma} .
$$

An application of Theorem 3.1 yields, for any $q>\sigma^{2} / 2+\delta$,

$$
\mathbb{E}\left[\left(\Sigma_{e_{q}}-K\right)_{+}\right]=\frac{\Gamma(b+1-\gamma)}{2^{\gamma} \Gamma(b+1)} \frac{1}{\gamma-1} K^{1-\gamma} \Phi\left(\gamma-1, b+1 ;-\frac{2}{K \sigma^{2}}\right) .
$$

Next, using the integral representation of the confluent hypergeometric function

$$
\Phi(a, b ; z)=\frac{\Gamma(b)}{\Gamma(a) \Gamma(b-a)} \int_{0}^{1} \mathrm{e}^{z t} t^{a-1}(1-t)^{b-a-1} \mathrm{~d} t, \quad \operatorname{Re}(b)>\operatorname{Re}(a)>0,
$$

we deduce that

$$
\begin{aligned}
\mathbb{E}\left[\left(\Sigma_{e_{q}}-K\right)_{+}\right] & =\left(\frac{2}{\sigma^{2}}\right)^{\gamma} \frac{K^{1-\gamma}}{\Gamma(\gamma)(b+1-\gamma)} \int_{0}^{1} \mathrm{e}^{-2 u / K \sigma^{2}} u^{\gamma-2}(1-u)^{b-\gamma+1} \mathrm{~d} u \\
& =\frac{2}{\sigma^{2}} \frac{1}{\Gamma(\gamma)(b+1-\gamma)} \int_{0}^{2 / K \sigma^{2}} \mathrm{e}^{-x} x^{\gamma-2}\left(1-\frac{K \sigma^{2}}{2} x\right)^{b-\gamma+1} \mathrm{~d} x,
\end{aligned}
$$

where we have performed the change of variable $x=2 u / K \sigma^{2}$. By choosing $\sigma=2$ and $\delta=2 v$, we recover the formula obtained by Geman and Yor [18, Formula (3.10)].

\subsection{The completely asymmetric tempered stable processes}

We now consider an example where the dynamics of the asset price are governed by a pure jump process. More specifically, we assume that $\xi$ is a spectrally negative tempered $\alpha$-stable Lévy process with $1<\alpha<2$. Its Laplace exponent admits the simple form

$$
\psi(u)=(\sigma u+\beta)^{\alpha}-\beta^{\alpha}, \quad u \geq 0,
$$


where the parameters $\sigma$ and $\beta$ are positive constants. We assume that $\sigma$ and $\beta$ are chosen such that $\psi(1)=r$, that is, $\sigma=\left(r+\beta^{\alpha}\right)^{1 / \alpha}-\beta$. These parametric Lévy processes are specific instances of the family of truncated Lévy processes constructed by Boyarchenko and Levendorskii [4]. Note that if $\beta=0$ then $\psi$ boils down to the Laplace exponent of a spectrally negative $\alpha$-stable Lévy process. Moreover, in the limit case $\alpha=2$, we recover the BlackScholes model with variance $2 \sigma^{2}$ and drift $2 \sigma \beta$. The Lévy measure of $\xi$ is absolutely continuous with a density, $v$, given by

$$
v(y)=C \frac{\mathrm{e}^{\beta y}}{|y|^{\alpha+1}}, \quad y<0,
$$

for some constant $C>0$. The inverse function of $\psi$ is $\phi(q)=\sigma^{-1}\left(q+\beta^{\alpha}\right)^{1 / \alpha}-\beta, q>0$, and

$$
\psi_{\gamma}(u)=\sigma^{\alpha}\left(\left(u+d^{1 / \alpha}\right)^{\alpha}-d\right),
$$

where $d=\left(\beta^{\alpha}+q\right) / \sigma^{\alpha}$. Note that $R=\infty$. Then

$$
a_{n}\left(\psi_{\gamma}\right)^{-1}=\sigma^{\alpha n} \prod_{k=1}^{n}\left(\left(k+d^{1 / \alpha}\right)^{\alpha}-d\right), \quad a_{0}\left(\psi_{\gamma}\right)=1 .
$$

Such an expression motivates us to introduce a generalization of the Pochhammer symbol, which is defined as $(z)_{\gamma}=\Gamma(z+\gamma) / \Gamma(z), \operatorname{Re}(z), \operatorname{Re}(\gamma)>0$. We define, for $n \in \mathbb{N}, \alpha>0$, and $z \in \mathbb{C}, \operatorname{Re}(z) \geq 0$,

$$
(z)_{n, \alpha}=\prod_{k=1}^{n}\left((k+z)^{\alpha}-z^{\alpha}\right) \quad \text { and } \quad(z)_{0, \alpha}=1 .
$$

Note the identities $(\beta)_{n, 1}=(\beta)_{n},(0)_{n, \alpha}=(1)_{n}^{\alpha}$, and $(\beta)_{n, 2}=(1)_{n}(2 \beta+1)_{n}$. Using this notation, for any $z, \rho \in \mathbb{C}$, we obtain the power series

$$
\iota_{\psi_{\gamma}}(\rho ; z)=\sum_{n=0}^{\infty} \frac{\Gamma(n+\rho)}{\left(d^{1 / \alpha}\right)_{n, \alpha} \Gamma(\rho)}\left(-\frac{z}{\sigma^{\alpha}}\right)^{n},
$$

which can be expressed in terms of the confluent hypergeometric function in the case $\alpha=2$. Finally, we obtain, with $C_{\gamma}$ given in (A.1) below,

$$
\mathbb{E}\left[\left(\Sigma_{e_{q}}-K\right)_{+}\right]=\frac{C_{\gamma}}{\gamma-1} K^{1-\gamma} \tau_{\psi_{\gamma}}\left(\gamma-1 ;-K^{-1}\right) .
$$

\subsection{The compound Poisson process with drift}

Finally, we consider as the last example a pure jump process but with finite activity. More precisely, we assume that the dynamics of $\xi$ are given, for any $t \geq 0$, by

$$
\xi_{t}=\delta t-\sum_{i=0}^{N_{t}} X_{i}
$$

where $\delta>0, N=\left(N_{t}\right)_{t \geq 0}$ is a Poisson process of parameter $p>0$, and the random variables $X_{0}, X_{1}, \ldots$ are independent and identically distributed with common distribution the exponential law of parameter $e>0$. The Laplace exponent of $\xi$ admits the form

$$
\psi(u)=u \frac{\delta u+\delta e-p}{u+e}, \quad u \geq 0 .
$$


It is easily seen that the condition $\delta=r+p /(1+e)$ gives $\psi(1)=r$. Moreover, a straightforward computation yields

$$
\phi(q)=\frac{1}{2 \delta}\left(\sqrt{4 e \delta q+(\delta e-p-q)^{2}}+q+p-\delta e\right), \quad q \geq 0,
$$

and

$$
\psi_{\gamma}(u)=\frac{\delta}{a} u \frac{u+b}{u+a},
$$

where $a=\gamma+e$ and $b=a-p e / \delta$. Thus,

$$
a_{n}\left(\psi_{\gamma}\right)=\left(\frac{a}{\delta}\right)^{n} \frac{\Gamma(n+a+1) \Gamma(b+1)}{\Gamma(n+b+1) \Gamma(n+1) \Gamma(a+1)}, \quad a_{0}\left(\psi_{\gamma}\right)=1,
$$

and, for $|z|<\delta / a$ and $\rho \in \mathbb{C}$, we have

$$
\begin{aligned}
\mathcal{I}_{\psi_{\gamma}}(\rho ; z) & =\frac{\Gamma(b+1)}{\Gamma(\rho) \Gamma(a+1)} \sum_{n=0}^{\infty} \frac{\Gamma(\rho+n) \Gamma(n+a+1)}{\Gamma(n+b+1) n !}\left(-\frac{a z}{\delta}\right)^{n} \\
& ={ }_{2} F_{1}\left(\rho, a+1 ; b+1 ;-\frac{a z}{\delta}\right),
\end{aligned}
$$

where ${ }_{2} F_{1}$ stands for the hypergeometric function; see [23, Chapter 9] for a detailed account on this function. Next, recalling the remarkable identity

$$
{ }_{2} F_{1}(-n, 1+a ; p ; 1)=\frac{\Gamma(b+1) \Gamma(n+1-p-a)}{\Gamma(b+1+n) \Gamma(1-p-a)},
$$

we recover from (2.5) the well-known Euler transformation

$$
{ }_{2} F_{1}(\rho, 1+a ; b+1 ; z)=(1-z)^{-\rho} F_{1}\left(\rho, 1-p-a ; p ; \frac{z}{z-1}\right), \quad|\arg (1-z)|<\pi,
$$

which provides an analytical continuation of the hypergeometric function into the half-plane $\operatorname{Re}(z)<\frac{1}{2}$. Then, using the asymptotic

$$
{ }_{2} F_{1}(\rho, 1+a ; b+1 ;-x) \sim \frac{\Gamma(b+1) \Gamma(a+1-\rho)}{\Gamma(b+1-\rho) \Gamma(a+1)} x^{-\rho} \quad \text { as } x \rightarrow \infty,
$$

we obtain

$$
C_{\gamma}=\frac{\Gamma(b+1-\gamma) \Gamma(a+1)}{\Gamma(b+1) \Gamma(a+1-\gamma)}=\frac{\Gamma(e(\delta-p) / \delta+1) \Gamma(\gamma+e+1)}{\Gamma(\gamma+e(\delta-p) / \delta+1) \Gamma(e+1)} .
$$

Finally, we obtain

$$
\mathbb{E}\left[\left(\Sigma_{e_{q}}-K\right)_{+}\right]=\frac{C_{\gamma}}{(\gamma-1)} K^{1-\gamma}{ }_{2} F_{1}\left(\gamma-1, \gamma+e+1 ; \gamma+\frac{e(\delta-p)}{\delta}+1 ;-\frac{a}{\delta K}\right) .
$$




\section{Appendix A. Some additional formulae}

We start the appendix by providing a contour integral representation of the function $I_{\psi_{\gamma}}$, which is found in [31, Proposition 2.4, Proposition 3.1]. To this end, we recall that $\gamma=\phi(q)$ and observe that, in the case $R=\infty$, as $0<\psi_{\gamma}^{\prime}\left(0^{+}\right)<\infty$, we have, for any $u>0$,

$$
\psi_{\gamma}(u)=\hat{\delta}_{\gamma} u+\frac{\sigma^{2}}{2} u^{2}+\int_{-\infty}^{0}\left(\mathrm{e}^{u y}-1-u y\right) \mathrm{e}^{\gamma y} m(\mathrm{~d} y)=u^{2} \bar{\varphi}_{\gamma}(u),
$$

where $\hat{\delta}_{\gamma}=\delta+\sigma \gamma+\int_{-\infty}^{0}\left(\mathrm{e}^{\gamma y}-\mathbf{1}_{\{|y|<1\}}\right) y m(\mathrm{~d} y)$ and

$$
\bar{\varphi}_{\gamma}(u)=\frac{\hat{\delta}_{\gamma}}{u}+\frac{\sigma^{2}}{2}+\int_{0}^{\infty} \mathrm{e}^{-u y} \int_{-\infty}^{-y} \int_{-\infty}^{-s} \mathrm{e}^{\gamma v} m(\mathrm{~d} v) \mathrm{d} s \mathrm{~d} y .
$$

Thus, one may define the function

$$
a_{s}\left(\psi_{\gamma}\right)=\frac{1}{\Gamma^{2}(s+1)} a_{s}\left(\bar{\varphi}_{\gamma}\right)=\frac{1}{\Gamma^{2}(s+1)} \prod_{k=1}^{\infty} \frac{\bar{\varphi}_{\gamma}(k+s+1)}{\bar{\varphi}_{\gamma}(k)}
$$

and observe the identity

$$
a_{s+1}\left(\bar{\varphi}_{\gamma}\right)=\frac{1}{\bar{\varphi}_{\gamma}(s+1)} a_{s}\left(\bar{\varphi}_{\gamma}\right)
$$

with $a_{0}\left(\bar{\varphi}_{\gamma}\right)=1$. Hence, $a_{s}\left(\bar{\varphi}_{\gamma}\right)$ is a meromorphic function in $F_{-\gamma}=\{z \in \mathbb{C} ; \operatorname{Re}(z)>-\gamma-1\}$ with simple poles at the points $z_{k}=-k-1$ for $k=0,1, \ldots$ and $z_{k}>-\gamma-1$.

Proposition A.1. Let us assume that $R=\bar{\delta}$. Then, for any $\rho \neq 0,-1, \ldots, \tau_{\psi_{\gamma}}(\rho ;$.) admits an analytical continuation in the entire complex plane cut along the positive real axis, given by

$$
\mathcal{I}_{\psi_{\gamma}}(\rho ; z)=\frac{1}{2 \mathrm{i} \pi \Gamma(\rho)} \int_{-\mathrm{i} \infty}^{\mathrm{i} \infty} a_{s}\left(\varphi_{\gamma}\right) \Gamma(s+\rho) \Gamma(-s) z^{s} \mathrm{~d} s, \quad|\arg (z)|<\pi,
$$

where the contour is indented to ensure that all poles (respectively nonnegative poles) of $\Gamma(s+\rho)$ (respectively $\Gamma(-s)$ ) lie to the left (respectively right) of the intended imaginary axis and, for any $\operatorname{Re}(s)>-1$,

$$
a_{s}\left(\varphi_{\gamma}\right)=\prod_{k=1}^{\infty} \frac{\varphi_{\gamma}(k+s+1)}{\varphi_{\gamma}(k)}
$$

with $\varphi_{\gamma}(s)=\bar{\delta}_{\gamma}-\hat{v}_{\gamma}(s)$ and $\hat{v}_{\gamma}(s)=\int_{0}^{\infty} \mathrm{e}^{-s r} \int_{-\infty}^{-r} \mathrm{e}^{\gamma v} m(\mathrm{~d} v) \mathrm{d} r$.

Otherwise, if $R=\infty$, using the same contour as above, we have

$$
\tau_{\psi_{\gamma}}(\rho ; z)=\frac{1}{2 \mathrm{i} \pi \Gamma(\rho)} \int_{-\mathrm{i} \infty}^{\mathrm{i} \infty} a_{s}\left(\bar{\varphi}_{\gamma}\right) \frac{\Gamma(s+\rho)}{\Gamma(s+1)} \Gamma(-s) z^{s} \mathrm{~d} s,
$$

which is valid in the sector $|\arg (z)|<\pi / 2$.

We now provide some representations of the constant appearing in the asymptotic (2.6) in terms of the Laplace exponent $\psi$. 
Proposition A.2. If $R=\bar{\delta}$ then

$$
C_{\gamma}=a_{-\gamma}\left(\varphi_{\gamma}\right)
$$

Otherwise, we have, writing $\psi(u)=u \varphi(u)$,

$$
C_{\gamma}= \begin{cases}\psi_{\gamma}^{\prime}\left(0^{+}\right) & \text {if } \gamma=1, \\ \psi_{\gamma}^{\prime}\left(0^{+}\right)\left(\prod_{k=1}^{n} \varphi(k)\right)^{-1} & \text { if } \gamma=n+1, n=1,2 \ldots, \\ \frac{1}{\Gamma(1-\gamma)} a_{-\gamma}\left(\bar{\varphi}_{\gamma}\right) & \text { otherwise. }\end{cases}
$$

\section{Acknowledgements}

I am indebted to the anonymous referee for very helpful and constructive suggestions. I am also grateful to D. Madan for bringing to my attention many relevant references and to F. Delbaen for many interesting discussions on the topic. Financial support from the National Bank of Belgium is gratefully acknowledged by the author.

\section{References}

[1] Albrecher, H., Dhaene, J., Goovaerts, M. and Schoutens, W. (2005). Static hedging of Asian options under Lévy models. J. Derivatives 12, 63-72.

[2] Bertoin, J. (1996). Lévy Processes. Cambridge University Press.

[3] Bertoin, J. And Yor, M. (2005). Exponential functionals of Lévy processes. Prob. Surveys 2, 191-212.

[4] Boyarchenko, S. I. And Levendorskit, S. Z. (2000). Option pricing for truncated Lévy processes. Internat. J. Theoret. Appl. Finance 3, 549-552.

[5] Carmona, P., Petit, F. And Yor, M. (1997). On the distribution and asymptotic results for exponential functionals of Lévy processes. In Exponential Functionals and Principal Values Related to Brownian Motion, ed. M. Yor, Rev. Mat. Iberoamericana, Madrid, pp. 73-130.

[6] Carmona, P., Petit, F. ANd Yor, M. (1998). Beta-gamma random variables and intertwining relations between certain Markov processes. Rev. Mat. Iberoamericana 14, 311-367.

[7] CARr, P. AND Schröder, M. (2003). Bessel processes, the integral of geometric Brownian motion, and Asian options. Teor. Veroyat. Primen. 48, 503-533. English translation: Theory Prob. Appl. 48 (2004), 400-425.

[8] Collin-Dufresne, P., Goldstein, R. S. and Yang, F. (2010). On the relative pricing of long maturity S\&P 500 index options and CDX tranches. NBER working paper 15734.

[9] Delbaen, F. and Schachermayer, W. (1994). A general version of the fundamental theorem of asset pricing. Math. Ann. 300, 463-520.

[10] Donati-Martin, C., Ghomrasni, R. And Yor, M. (2001). On certain Markov processes attached to exponential functionals of Brownian motion; application to Asian options. Rev. Mat. Iberoamericana 17, 179-193.

[11] Dufresne, D. (1990). The distribution of a perpetuity, with applications to risk theory and pension funding. Scand. Actuarial J. 1990, 39-79.

[12] Dufresne, D. (2000). Laguerre series for Asian and other options. Math. Finance 10, 407-428.

[13] Eberlein, E. and Papapantoleon, A. (2005). Equivalence of floating and fixed strike Asian and lookback options. Stoch. Process. Appl. 115, 31-40.

[14] Eberlein, E. ANd Madan, D. B. (2010). Short positions, rally fears and option markets. Appl. Math. Finance 17, 83-98.

[15] Eberlein, E., JaCod, J. AND Raible, S. (2005). Lévy term structure models: no-arbitrage and completeness. Finance Stoch. 9, 67-88.

[16] Fu, M. C., Madan, D. B. And Wang, T. W. (1999). Pricing continuous Asian options: a comparison of Monte Carlo and Laplace transform inversion methods. J. Comput. Finance 2, 49-74.

[17] Geman, H. and Yor, M. (1992). Quelques relations entre processus de Bessel, options asiatiques et fonctions confluentes hypergéométriques. C. R. Acad. Sci. Paris 314, 471-474.

[18] Geman, H. and Yor, M. (1993). Bessel processes, Asian options, and perpetuities. Math. Finance 3, 349-375.

[19] Gjessing, H. and Paulsen, J. (1997). Present value distributions with applications to ruin theory and stochastic equations. Stoch. Process. Appl. 71, 123-144.

[20] Gradshteyn, I. S. And Ryshik, I. M. (2000). Table of Integrals, Series, and Products, 6th edn. Academic Press, San Diego, CA. 
[21] Henderson, V. and Wojakowski, R. (2002). On the equivalence of floating- and fixed-strike Asian options. J. Appl. Prob. 39, 391-394.

[22] Kyprianou, A. E. (2006). Introductory Lectures on Fluctuations of Lévy Processes with Applications. Springer, Berlin.

[23] Lebedev, N. (1972). Special Functions and Their Applications. Dover Publications, New York.

[24] Linetsky, V. (2004). The spectral decomposition of the option value. Internat. J. Theoret. Appl. Finance 7, 337-384.

[25] Madan, D. And Schoutens, W. (2008). Break on through to the single side. Working paper, Katholieke Universiteit Leuven.

[26] Maulik, K. and Zwart, B. (2006). Tail asymptotics for exponential functionals of Lévy processes. Stoch. Process. Appl. 116, 156-177.

[27] Olver, F. W. J. (1974). Asymptotics and Special Functions. Academic Press, New York.

[28] Patie, P. (2008). $q$-invariant functions for some generalizations of the Ornstein-Uhlenbeck semigroup. ALEA Lat. Amer. J. Prob. Math. Statist. 4, 31-43.

[29] Patie, P. (2009). Infinite divisibility of solutions to some self-similar integro-differential equations and exponential functionals of Lévy processes. Ann. Inst. H. Poincaré Prob. Statist. 45, 667-684.

[30] Patie, P. (2009). Law of the exponential functional of one-sided Lévy processes and Asian options. C. R. Acad. Sci. Paris 347, 407-411.

[31] Patie, P. (2012). Law of the absorption time of some positive self-similar Markov processes. Ann. Prob. 40, 765-787.

[32] Rogers, L. C. G. And Shi, Z. (1995). The value of an Asian option. J. Appl. Prob. 32, 1077-1088.

[33] Schoutens, W. (2003). Lévy Processes in Finance. Pricing Finance Derivatives. John Wiley, New York.

[34] SCHRÖDER, M. (2005). Laguerre series in contingent claim valuation, with applications to Asian options. Math. Finance 15, 491-531.

[35] SCHRÖDER, M. (2008). On constructive complex analysis in finance: explicit formulas for Asian options. Quart. Appl. Math. 66, 633-658.

[36] VEČĚ̌, J. AND Xu, M. (2004). Pricing Asian options in a semimartingale model. Quant. Finance 4, 170-175.

[37] Yor, M. (2001). Exponential Functionals of Brownian Motion and Related Processes. Springer, Berlin. 